\title{
Development of a mathematical model for simultaneous heave, pitch and roll motions of planing vessel in regular waves
}

\author{
Parviz Ghadimi*, Abbas Dashtimanesh,Seyed Reza Djeddi,Yaser Faghfoor Maghrebi \\ Department of Marine Technology, Amirkabir University of Technology, Tehran, Iran \\ *Corresponding authorE-mail: pghadimi@aut.ac.ir
}

\begin{abstract}
The market for planing boats has shown steady growth in the recent years and this has caused an increase in the need for designing tools. The need is even more vital for the prediction of seakeeping and maneuverability of planing boats. Although the numerical methods are proved to be useful for the displacement vessels, they seem to be relatively impractical dealing with the complexities of the hydrodynamic behavior of planing boats. In this paper, a simple method for prediction of Heave, Pitch and Roll motions of the planing vessels in regular waves is presented. In the formulation of the present mathematical model, a 2-dimensional technique using momentum theory is applied. Different aspects of the added mass and 2D+t theories are also implemented for the section-based simulations of the planing vessel. The developed method is analyzed through a set of validation tests while the experimental results of the literature are used in order to validate the obtained results. The results are then evaluated in different degrees of freedom and favorable agreement has been achieved against the experimental data.
\end{abstract}

Keywords: Planing Boat; Heave; Pitch; Roll; Regular Wave; Mathematical Model

\section{Nomenclature}

$m$ : Mass

$x_{C G}, z_{C G}$ : Position of Center of Gravity (COG) of the hull in $\mathrm{x}$ and $\mathrm{z}$ directions

$\dot{x}_{C G}, \dot{z}_{C G}:$ Velocity at COG in $\mathrm{x}$ and $\mathrm{z}$ directions

$\ddot{x}_{C G}, \ddot{z}_{C G}:$ Acceleration at COG in $\mathrm{x}$ and $\mathrm{z}$ directions

$F_{x}, F_{z}:$ Force in $\mathrm{x}$ and $\mathrm{z}$ directions

$X_{r}, Z_{r}:$ Hydrostatic force in $\mathrm{x}$ and $\mathrm{z}$ directions

$X_{w}, Z_{w}:$ Hydrodynamic force in $\mathrm{x}$ and $\mathrm{z}$ directions

$X_{f}, Z_{f}:$ Wave force in $\mathrm{x}$ and $\mathrm{z}$ directions

$I_{G x x}, I_{G y y}:$ Moment of Inertia in xx and yy directions

$\psi:$ Roll angle

$\dot{\psi}$ : Angular velocity of Roll motion

$\ddot{\psi}$ : Acceleration of roll motion

$\theta$ : Pitch angle

$\dot{\theta}$ : Angular velocity of pitch motion

$\ddot{\theta}$ : Acceleration of pitch motion

$M_{x x}, M_{y y}:$ Moment in xx and yy directions.

$K_{r}, P_{r}, R_{r}$ : Hydrostatic moment in xx, yy and zz directions

$K_{w}, P_{w}, R_{w}:$ Hydrodynamic moment in xx, yy and zz directions 
$K_{f}, P_{f}, R_{f}:$ Wave moment in xx, yy and zz directions

$U:$ Velocity component parallel to the keel

$(\zeta, \xi):$ Coordinate system on the hull

$t$ : Time

$w_{z}$ : Vertical component of the wave orbital velocity at the surface

$\dot{w}_{z}:$ Time derivative of $w_{z}$

$V$ : Normal velocity

$\dot{V}:$ Time derivative of normal velocity

$a$ : Wave amplitude

$k$ : Wave number

$\omega_{e}:$ Encounter frequency

$\varepsilon$ : Phase angle

$f_{M}$ : Hydrodynamic lift force associated with the change of fluid momentum per unit length

$f_{M P T}: f_{M}$ associated with port side

$f_{M S B}: f_{M}$ associated with starboard side

$m_{a}:$ Added mass

$m_{a_{P T}}:$ Added mass associated with port side

$m_{a_{S B}}$ : Added mass associated with starboard side

$\dot{m}_{a}:$ time derivative of added mass

$k_{a}:$ added mass coefficient

$\rho:$ Water density

$b$ : instantaneous half beam of the section

$\dot{b}$ : time derivative of $b$

$d$ : Depth of penetration

$\beta$ : Deadrise angle

$d_{e}:$ Effective depth of penetration

$C_{p u}:$ splash up coefficient

$h:$ Submergence of a section

$\eta$ : Wave height

$v$ : Wave slope

$C_{D}$ : Drag coefficient

$f_{C D}$ : Viscous lift force associated with the cross flow drag

$a_{B F}:$ Buoyancy coefficient

\section{Introduction}

The sea-keeping of vessels can be predicted using the investigation of accelerations and behaviors of their Roll motion. Additionally, passengers' comfort and the suitable operation of ship structure and machineries are of high importance. Therefore, prediction of ship performance is the key to a successful design. A multitude of theoretical and mathematical studies can be traced in the literature, during the past decades.

The difficulties and complexities of the hydrodynamic behavior of planing vessels have been addressed since this type of boat became popular with the pioneer work being done by Faltinsen [1]. At higher speeds, the planing condition exhibits highly non-linear phenomena that have never been seen in displacement vessels. These non-linearities lead to excessive 
instabilities [2].

Dynamic pressure dominance, changes in the wetted length, spray effect, considerable changes in the rising of the vessel and its trim angle are all among the factors that should be considered in accurate modeling of planing boats. Consequently, many theoretical and experimental research activities have been done on the analysis of stable ship motions in calm waters $[3,4]$. For unstable condition of planing vessel motion in waves, use of experimental data has been proved to be a reliable method. Also, a number of analytical methods have been introduced and developed in recent years both in frequency and time domains $[5,6,7]$. Methods based on the $2 \mathrm{D}+\mathrm{t}$ theory have gained higher attentions and they have been improved by different authors [8,9] while Garme [10] has claimed the development of a reliable procedure as an effective tool for prediction of ship behaviors. Due to the fact the $2 \mathrm{D}+\mathrm{t}$ method has been studied and validated in wide ranges of applications, it can be used as a favorable method for designers and researchers while giving time-dependant results and assuring acceptable computational accuracy.

In the present paper, based on the method introduced by Payne [11], a mathematical model for studying Roll motions in ships has been developed. In the framework of this study, instead of considering a symmetric water impact of the section using 2D+t theory, the unsymmetrical impact has been studied. Additionally, in order to take the wave effects into considerations, regular wave theory is used. Moreover, momentum and added mass theories have been applied for the evaluation of forces acting on the 2-dimensional sections.

In the following section, the developed method is presented and discussed in depth and after the validation of the method's fundamentals, the results for the Roll, Heave and Pitch coupled motions are provided. As a matter of fact, the present study is a primary step toward the development and implementation of mathematical models for higher degrees of freedom (DOFs). In this regard, using simplifying assumptions can be reasonable to some extent.

\section{Coordinate system definition}

Due to the highly non-linear physics of planing vessels, the combination of different motions in various degrees of freedom would be a normal approach. In this regard, the Heave, Pitch and Roll motions of ships have attracted the attention of many scientists. In addition to this, there would be no use in studying these effects without taking the wave effects into consideration. Generally in this paper, the three degrees of freedom (3DOF) dynamic of a planing ship with prismatic body in regular waves is studied.

For the development of the mathematical model, two coordinate systems are introduced; one being placed on undisturbed free-surface and the other on the vessel's center of gravity. The Oxyz coordinate system with the origin of $O$ is placed on the calm water line WL (Figs.1 and 2). The X-axis is on the symmetric plane of the ship and the z-axis is vertical with its positive region pointing downward. The other coordinate system is assumed to be on the body and is free to move with the ship (Figs. 1 and 2).

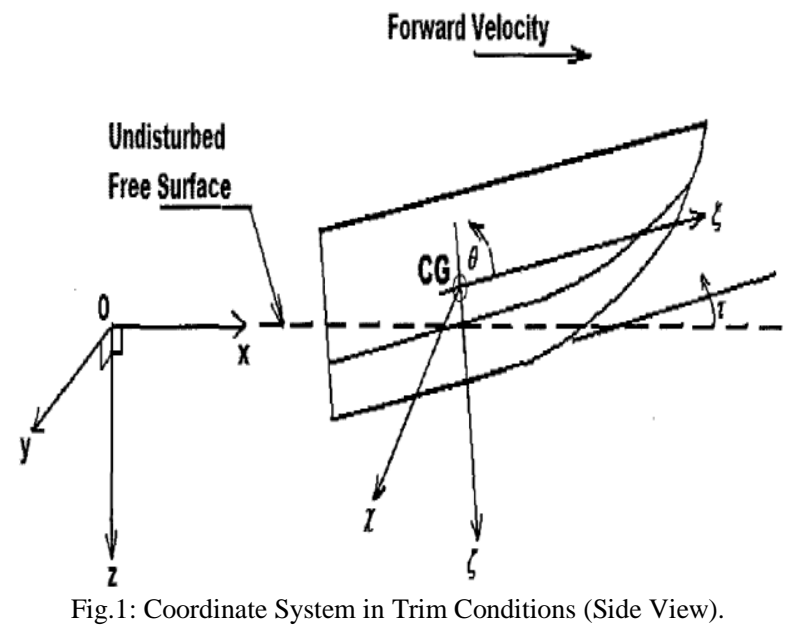




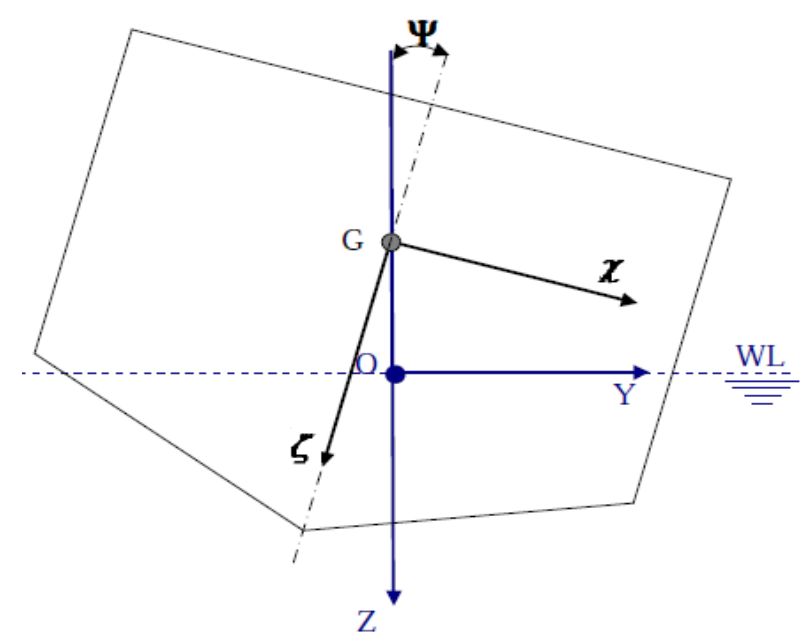

Fig.2: Coordinate System in Roll Conditions (Front View).

\section{Governing equations}

Consider the fixed coordinate systems with its $\mathrm{x}$-axis placed on undisturbed free-surface and its $\mathrm{z}$-axis pointing downward. If the ship motion is limited to Pitch $\theta$, Heave $z_{C G}$, Surge $x_{C G}$ and Roll $\psi$, then the equation of motion are written as follows (subscripts $r, w$ and $f$ refer to hydrostatic, wave and hydrodynamics terms):

$$
\begin{aligned}
& m . \ddot{x}=F_{x}(t)=X_{r}(t)+X_{w}(t)+X_{f}(t) \\
& m . \ddot{z}=F_{z}(t)=Z_{r}(t)+Z_{w}(t)+Z_{f}(t) \\
& I_{G x x} \cdot \ddot{\psi}=M_{x}(t)=K_{r}(t)+K_{w}(t)+K_{f}(t) \\
& I_{G y y} \cdot \ddot{\theta}=M_{y}(t)=P_{r}(t)+P_{w}(t)+P_{f}(t)
\end{aligned}
$$

In which $\mathrm{m}$ is the mass and $\mathrm{I}$ is the inertial moment of the ship. $\mathrm{F}$ and $\mathrm{M}$ also refer to Force and Moment terms acting on the ship.

\section{$4 \quad 2 D+t$ Theory}

For a ship with prismatic body, due to the fact that the sections are similar length-wise, the analysis of the ship motion is identical to the water entry problem of the wedge with velocity V. As shown on Fig.3, the wedge initially touches the water surface at $t=t_{0}$. Then, an instantaneous boundary value problem is solved on the control surface. In fact, a 2-dimensional problem is studied using 3-dimensional boundary conditions.

Generally, a planing vessel with prismatic body can be assumed with trim angle of $\tau$ and advancing velocity of $U$. Fig.3 shows how it is possible to use a $2 \mathrm{D}$ analysis method to have a $2 \mathrm{D}+\mathrm{t}$ analysis of the steady flow in reference to a fixed coordinate on the ship.

Moreover, an inclining moment that is generated due to various factors, is introduced and discussed (Fig.4). Generally, this can be considered as a development in $2 \mathrm{D}+\mathrm{t}$ theory. In all of the previous studies, only heave and pitch motions have been investigated while in the current article roll motion is also included. 

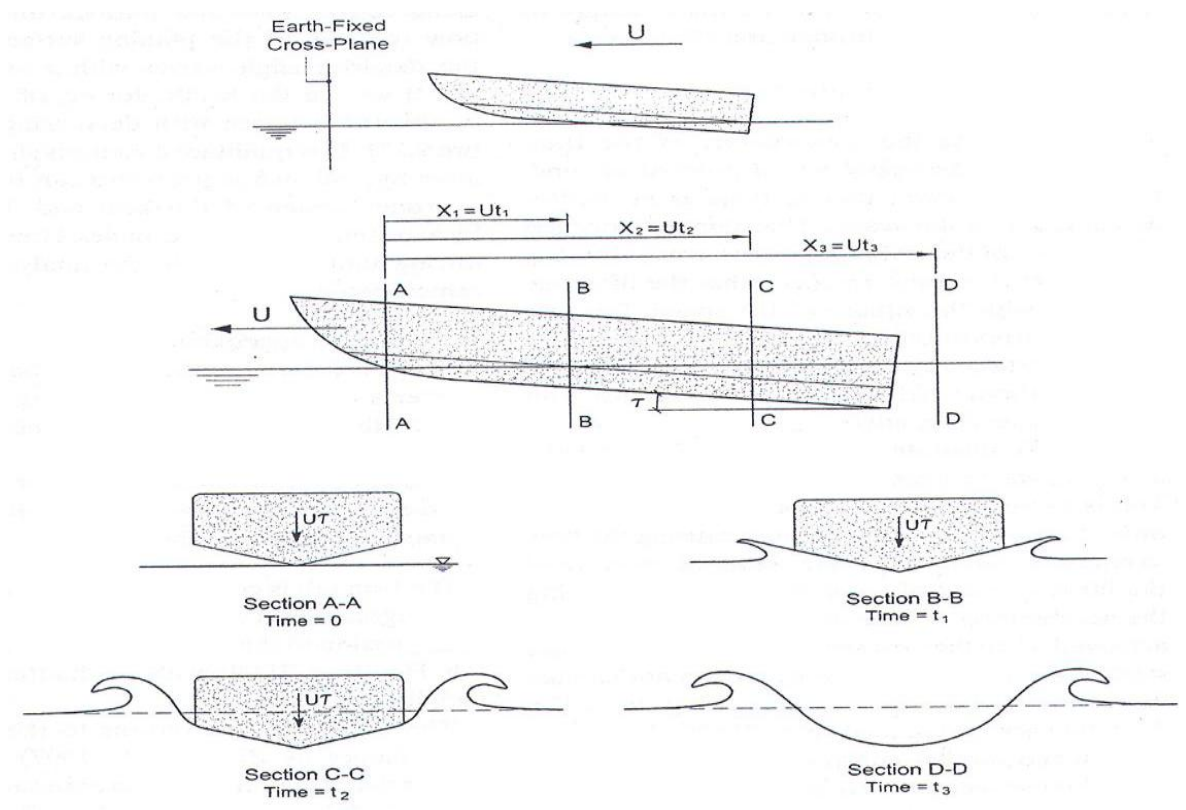

Fig.3: 2D+t Theory.

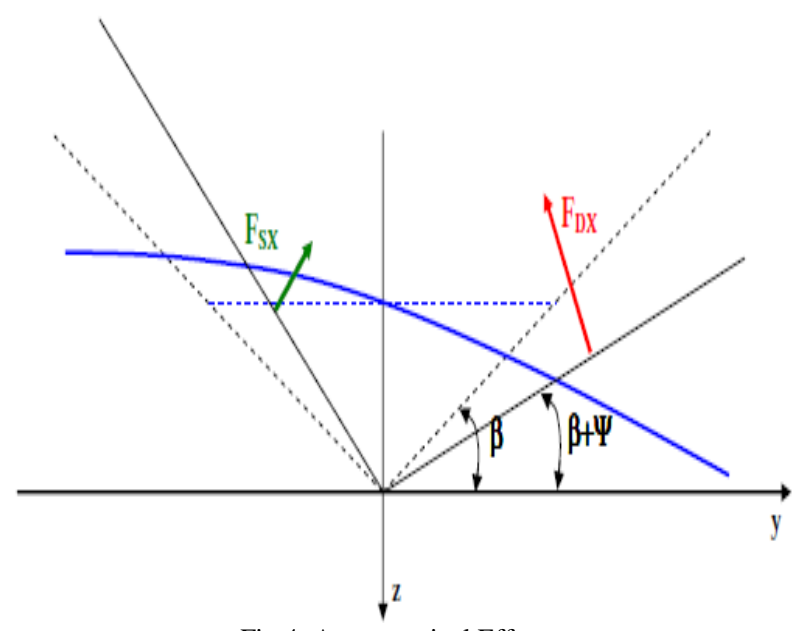

Fig.4: Asymmetrical Effects.

In particular, three major effects are taken into account:

1) Asymmetrical force of fluid acting on the body: The vessel in its inclined condition is exposed to different forces acting on its starboard and port sides which are the result of absolute wave velocities and ship motions relative to Roll.

2) Asymmetrical wedge water impact: As a result of Roll motion, the section impacts the water asymmetrically. The starboard and port sides are considered separately based on their respective dead-rise angles. These angles come from the local geometrical and Roll angles that are used for the calculation of added mass terms.

3) Asymmetrical submerged geometrical volume: The submerged volume and the wetted surface are often not symmetrical and this would affect the location on which the hydrostatic and other forces will play a part.

As a final result, the side forces acting on starboard and port sides are calculated separately and for each section. This means that an asymmetrical water impact instead of a symmetrical one should be considered for the $2 \mathrm{D}+\mathrm{t}$ theory. In addition to this, the assumption for the separate calculation of forces acting on either sides of the vessel should be established prior to the estimation of hydrodynamic coefficients and forcing terms. As a matter of fact, it should be proved that when the Roll motion occurs and the body has a dead-rise angle of $\beta$ on one side, the force acting on this side of the body is identical to the forces acting on a symmetrical body with a dead-rise angle of $\beta$. In this regard, the analytical method of Toyama [12] which is developed based on the Wagner Method [13] for asymmetrical bodies, is utilized.

The pressure distribution over the wedge body due to the water impact in symmetrical and asymmetrical conditions can now be calculated using the Toyama method. The pressure distribution for wedges with dead-rise angles of 10 and 20 degrees are depicted in Figs.5 and 6. It should be noted that the wedge has the dead-rise angle of 10 or 20 degrees on one 
side and the angle on the other side is set to be variable.

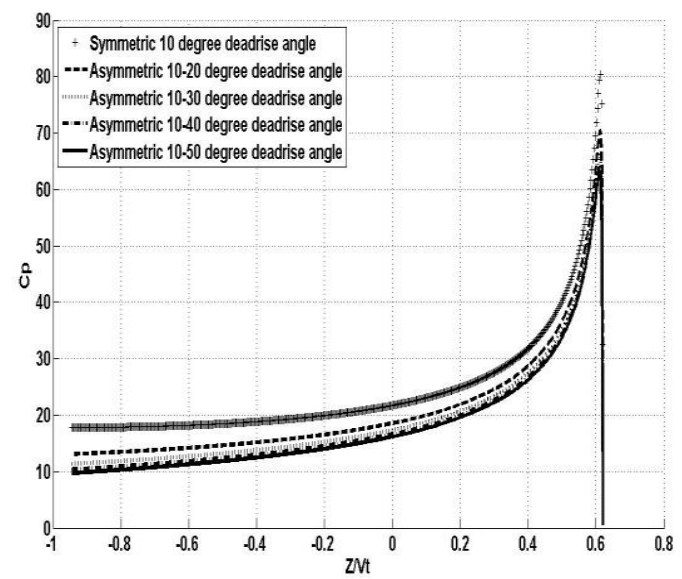

Fig.5: Investigation of asymmetry for a wedge with 10 degrees deadrise angle [12].

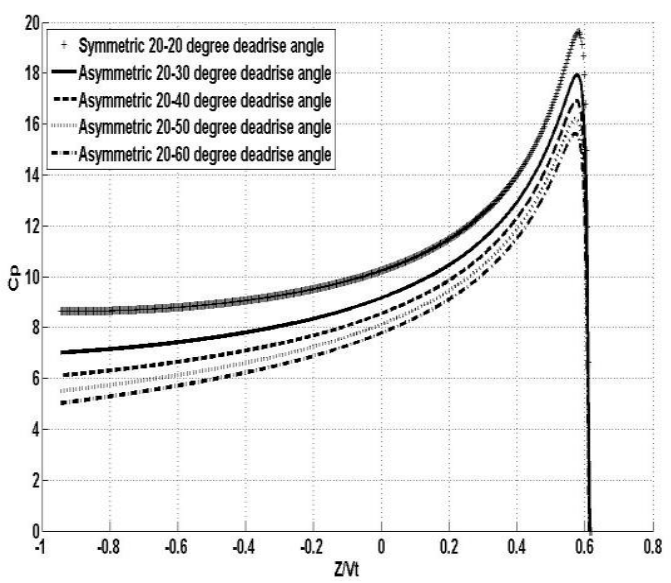

Fig.6: Investigation of asymmetry for a wedge with 20 degrees deadrise angle [12].

Based on the results of the present method and the ones from [14], it is clear that the separately calculated forces on two sides of the wedge are approximately reasonable and acceptable. Now, it is possible to calculate hydrodynamic coefficients and forcing loads on the body section with the present method.

\section{Momentum theory}

The forcing loads on the ship section are shown on Fig. 5 and include 5 terms, i.e. section weight (w), Hydrodynamic lift force due to momentum changes in fluid $\left(f_{M}\right)$, Viscous lift force due to cross-flow drag forces $\left(f_{C D}\right.$ ) and a buoyancy force due to the instantaneous displacements $\left(\mathrm{f}_{\mathrm{B}}\right)$.

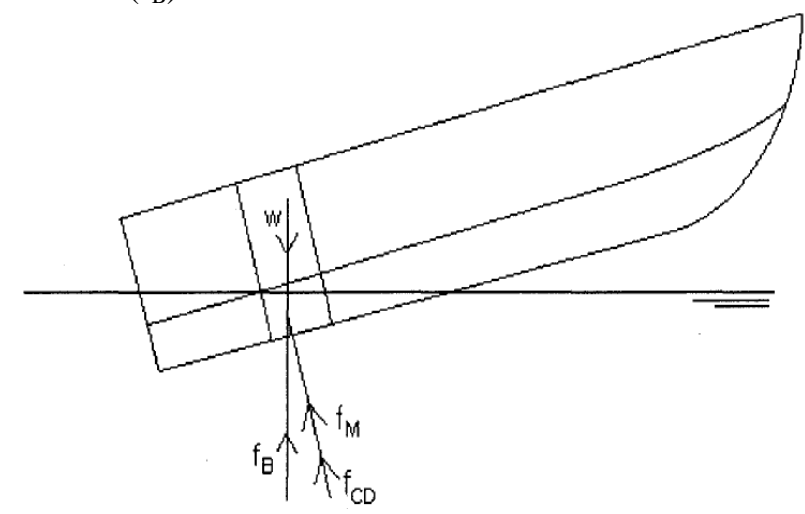

Fig.7: Forces acting on a ship section. 
The hydrodynamic lift force due to the momentum changes in fluid per unit length that acts on the section is evaluated as follows [6]:

$$
\begin{aligned}
f_{M} & =\frac{D}{D t}\left(m_{a} V\right)=m_{a} \dot{V}+V \dot{m}_{a}-\frac{\partial}{\partial \xi}\left(m_{a} V\right) \frac{d \xi}{d t} \\
& =m_{a} \dot{V}+V \dot{m}_{a}-U \frac{\partial}{\partial \xi}\left(m_{a} V\right)
\end{aligned}
$$

Where $m_{a}$ is the added mass of the section, $\mathrm{U}$ is the relative fluid velocity parallel to the keel and $\mathrm{V}$ is the velocity in the plane of the section and perpendicular to the reference line.

A similar equation can be used based on the hydrodynamic forces on either sides of the section in order to evaluate the added mass terms.

$$
\begin{aligned}
& f_{M P T}=-\frac{D}{D t}\left(m_{A_{P T}} \cdot{ }^{v} n_{\text {rel } P T}\right) \\
& f_{M S B}=-\frac{D}{D t}\left(m_{A_{S B}} \cdot n_{n_{\text {rel } S B}}\right)
\end{aligned}
$$

Moreover, the lift force due to the cross-flow drag per unit length is expressed as below [6]:

$$
f_{C D}=C_{D} \rho b V^{2}
$$

Where $C_{D}$ the drag coefficient of the cross-flow is, $\rho$ is the fluid density and b is the half-breadth.

The buoyancy force per unit length is then generated as follows [6]:

$$
f_{B}=a_{B F} \rho g A
$$

In which $a_{B F}$ is the buoyancy correction coefficient and $\mathrm{A}$ is the transverse section area.

It is assumed that the flow in transverse sections is perpendicular to the keel and is not affected by the cross-flows in different longitudinal sections. Therefore, two conditions are expected; first, the condition in which the chine remains dry that happens close to the edge of the vessel's wetted length and second is when the chine in vicinity of the transom stern gets wet. The sections expanding in the middle of the bow and stern parts would oscillate between these two conditions.

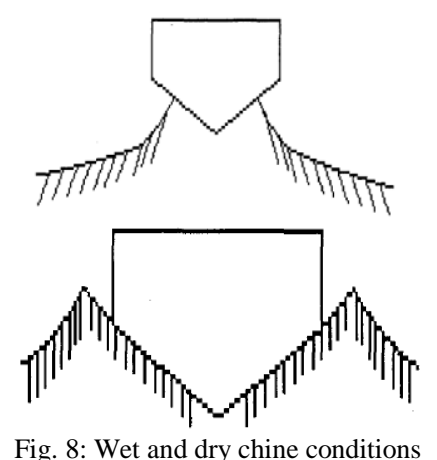

\section{$6 \quad$ Added mass theory}

The added mass refers to a concept that is widely used for the sea-keeping, maneuverability and planing calculations. The value of the added mass changes based on the shape and dimension of the body. The added mass for a wedge-shaped section is calculated using the following formulas [6]:

$$
\begin{aligned}
& \qquad m_{a}=k_{a} \frac{\pi}{2} \rho b^{2} \\
& \dot{m}_{a} \approx k_{a} \pi \rho b\left(C_{p u} \cot \beta\right) \frac{\dot{z}-\dot{\eta}}{\cos \theta-v \sin \theta} \\
& \text { In which v is the local wave slope, } C_{p u} \text { is the spray coefficient and } k_{a} \text { is a constant factor. }
\end{aligned}
$$




\section{Hydrodynamic forces and moments}

\subsection{Vertical Forces}

The vertical forces are calculated similar to the axial and side forces. The only difference is in the projection of the force components on the vertical direction. Based on the force-body diagram, it is known that Finally, the force equation in vertical direction can be written as follows:

$$
\begin{aligned}
& F_{z}=\left(\int_{l} f_{M} d \xi-\int_{l} f_{C D} d \xi\right) \cos \psi \cos \theta-\int_{l} f_{B} d \xi \\
& =\left(\int_{l}\left(N_{M_{P}}+N_{M_{S}}\right) d \xi-\int_{l}\left(f_{C D_{P}}+f_{C D_{S}}\right) d \xi\right) \cos \psi \cos \theta-\int_{l} a_{B F} \rho g A d \xi \\
& =\left(\int_{l}\left(\frac{D}{D t}\left(m_{a_{P}} V_{P}\right)+\frac{D}{D t}\left(m_{a_{S}} V_{S}\right)\right) d \xi-\int_{l}\left(f_{C D_{P}}+f_{C D_{S}}\right) d \xi\right) \cos \psi \cos \theta-\int_{l} a_{B F} \rho g A d \xi \\
& =-\left[\int_{l}\left(m_{a_{P}} \dot{V_{P}}+m_{a_{S}} \dot{V_{S}}+\dot{m}_{a_{P}} V_{P}+\dot{m}_{a_{S}} V_{S}-U_{P} \frac{\partial}{\partial \xi}\left(m_{a_{P}} V_{P}\right)-U_{S} \frac{\partial}{\partial \xi}\left(m_{a_{S}} V_{S}\right)\right) d \xi\right. \\
& \left.+\int_{l}\left(C_{D} \rho\left(b_{P} V_{P}^{2}+b_{S} V_{S}^{2}\right)\right) d \xi\right] \cos \psi \cos \theta-\int_{l} a_{B F} \rho g A d \xi \\
& F_{z}=\left[-M_{a_{P}} \ddot{x}_{C G} \sin \theta+Q_{a_{P}} \ddot{\theta}-M_{a_{P}} \ddot{z}_{C G} \cos \theta+\int_{l} m_{a_{P}}\left(\frac{d w_{z}}{d t}+U \frac{\partial w z_{P}}{\partial \xi}\right) \cos \theta d \xi\right. \\
& -M_{a_{P}} \dot{\theta}\left(\dot{x}_{C G} \cos \theta-\dot{z}_{C G} \sin \theta\right)-\int_{l} m_{a_{P}{ }^{w} z_{P}} \dot{\theta} \sin \theta d \xi-\int_{l} \dot{m}_{a_{P}} V_{P} d \xi+\left.U_{P} V_{P} m_{a_{P}}\right|_{b o w} ^{\text {stern }} \\
& \left.-\int_{l} m_{a_{P}} V_{P} \frac{\partial U}{\partial \xi} d \xi-\int_{l} C_{D} \rho b_{P} V_{P}^{2} d \xi+\{\text { Similar S terms }\}\right] \cdot \cos \theta \cdot \sin \psi-\int_{l} a_{B F} \rho g A d \xi
\end{aligned}
$$

\subsection{Pitch moment equation}

The moment that causes Pitch motion can also be evaluated using Heave force. In fact, with the calculation of force components, evaluation of the moment would be straight-forward. However, it is required to calculate the effecting point of these forces. There are two methods for calculation of the moment.

\subsubsection{First method}

Pitch moment can only be affected by the total vertical forces acting on the ship. Therefore we have:

$$
M_{y y}=\left(F_{z_{P}} x_{c_{P}}+F_{z_{S}} x_{c_{S}}\right)=F_{z} x_{c}
$$

Where $F_{z_{P}}$ and $F_{z_{S}}$ are the vertical forces on the starboard and port sides which cause Pitch moment.

\subsubsection{Second Method}

In the second method, moment arm is separately multiplied by the forces on each side. Therefore, we have: 


$$
\begin{aligned}
& M_{y y}=\left[Q_{a_{P}} \ddot{x}_{C G} \sin \theta-I_{a_{P}} \ddot{\theta}+Q_{a_{P}} \ddot{z}_{C G} \cos \theta-\int_{l} m_{a_{P}}\left(\frac{d w z_{P}}{d t}+U \frac{\partial w{ }^{w}}{\partial \xi}\right) \cos \theta \xi d \xi\right. \\
& -Q_{a_{P}} \dot{\theta}\left(\dot{x}_{C G} \cos \theta-\dot{z}_{C G} \sin \theta\right)+\int_{l} m_{a_{P}{ }^{w} z_{P}} \dot{\theta} \sin \theta \xi d \xi+\int_{l} \dot{m}_{a_{P}} V_{P} \xi d \xi \\
& +\left.U_{P} V_{P} m_{a_{P}} \xi\right|_{\text {stern }}+\int_{l} m_{a_{P}} V_{P} \frac{\partial w}{\partial \xi} \sin \theta \xi d \xi+\int_{l} C_{D} \rho b_{P} V_{P}^{2} \xi d \xi \\
& +\{\text { Similar } S \text { terms }\}] \cdot \cos \psi-\int_{l} a_{B F} \rho g A \cos \theta \xi d \xi
\end{aligned}
$$

Here, the second method is used.

\subsection{Roll moment equation}

When all the force components in axial, transverse and vertical directions are calculated, the moments acting on the ship can be easily found for different directions. In this section, Roll motion moment, which is due to the forces in transverse and vertical directions, is studied.

The side forces on either sides of the body have different effecting points in vertical direction. Moreover, the vertical forces on each side can have different values and asymmetric effecting points. All three factors pointed out here would cause Roll moment. Therefore, it can be written

$$
M_{x x}=\left(F_{y_{P}}-F_{y_{S}}\right) z_{c}+\left(F_{z_{P}} y_{P}-F_{z_{S}} y_{S}\right)
$$

In which $M_{x x}$ is the Roll moment and $F_{y_{P}}, F_{y_{S}}, F_{z_{P}}$ and $F_{z_{S}}$ are transverse and vertical forces on each side of the body which have been calculated previously. The only remaining part is the effecting point of the above mentioned forces which can be evaluated by integration.

\section{Regular wave model}

All the assumptions that have been previously used by Zarnick [6] are implemented in the present study. The length-wise $\mathrm{U}$ velocity and the $\mathrm{V}$ velocity which is perpendicular to the reference line of the ship, can be formulated as follows:

$$
\begin{aligned}
& U(\xi, t)=\dot{x}_{C G}(t) \cos \theta(t)-\left[\dot{z}_{C G}(t)-w_{z}(\xi, t)\right] \sin \theta(t) \\
& V(\xi, t)=\dot{x}_{C G}(t) \sin \theta(t)-\left[\dot{z}_{C G}(t)-w_{z}(\xi, t)\right] \cos \theta(t)-\dot{\theta}(t) \xi
\end{aligned}
$$

Where $\mathrm{w}_{\mathrm{z}}$ is the vertical component of the wave's orbital velocity.

The time-dependant submerged depth (h) for the transverse section on each point $(P(\xi, \zeta))$ is calculated as follows [6]:

$$
h(\xi, \zeta, t)=Z_{C G}(t)-\xi \sin \theta(t)+\zeta \cos \theta(t)-r(\xi, t)
$$

In which $r(\xi, t)$ is the instantaneous wave height on point $P(\xi, \zeta)$.

\section{Solution of the governing equations}

The analytical solution of equations of motion is very tedious and exhausting. However, using standard numerical techniques, the solution of the governing equations can be feasible. With the introduction of state vectors, equations of motion can be rewritten as follows:

$$
A \overrightarrow{\dot{x}}=\vec{g} \rightarrow \overrightarrow{\dot{x}}=A^{-1} \vec{g}
$$

Where $A^{-1}$ is inverse of matrix $A$. Here, the above equation is numerically integrated and solved using Runge-Kutta-Merson method. 


\section{Results}

Due to the fact that analytical, numerical and experimental results are not available to be used for validation of planning vessel's Roll motion, the presently developed method is validated against the governing mathematical basis of the problem at hand. The experiments of Fridsma [15] in regular waves have been done on planning vessels with the dead-rise angle of 20 degrees (Fig.9 and Table 1). The conditions of the experiments on Model A are presented in Table 2. Different conditions for Model A are studied using the present method and the results are depicted on Fig.10.
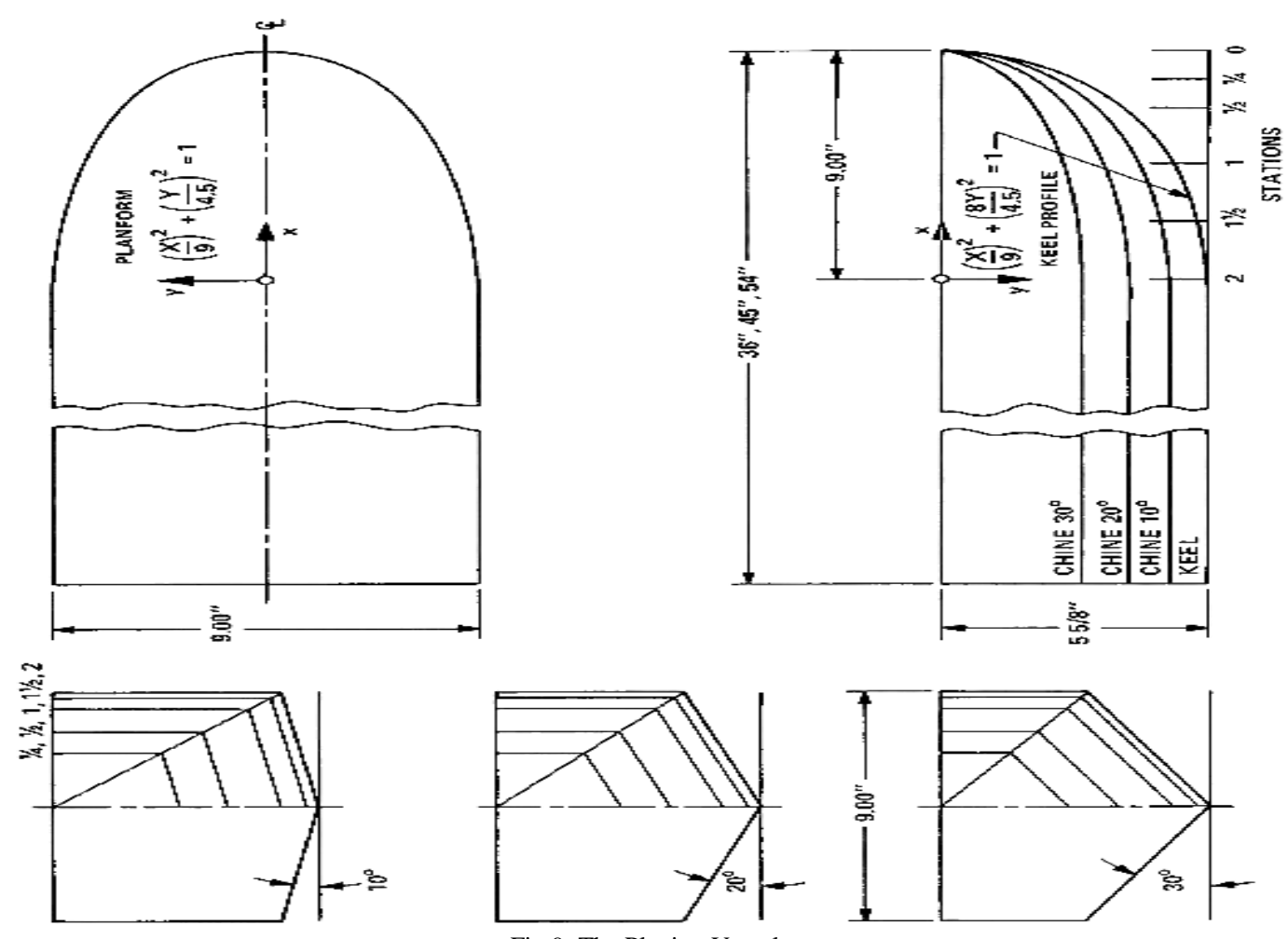

Fig.9: The Planing Vessel.

Table 1: The specification of the model used in Fridsma's experiments [15].

\begin{tabular}{ccc}
\hline Model & A & B \\
\hline $\mathrm{L} / \mathrm{b}$ & 5 & 5 \\
$\beta(\mathrm{deg})$ & 20 & 20 \\
$\mathrm{LCG}(\% \mathrm{~L})$ & 59 & 62 \\
$\tau(\operatorname{deg})$ & 4 & 4 \\
$\mathrm{~V} / \sqrt{\mathrm{L}}$ & 4 & 6 \\
$\mathrm{C}_{\Delta}$ & 0.608 & 0.608 \\
\hline
\end{tabular}

In Fig.10, the results of Heave and Pitch motions of Model A in different wave lengths are presented. As seen in the figure, the results of the developed software in longer waves are in better agreement with the experimental results. This is due to the assumption of wave lengths in the developed mathematical model which are assumed to be adequately longer than the ship's length. However, the obtained results are favorable for the initial designs with a reasonable error.

In addition to this, the reason for the deviations between the present results and those of experiments can be sought in the limitation of the mathematical model and the simplifications used. However, the accuracy of the results can be improved with the addition of the empirical and semi-empirical coefficients to the developed equations.

In addition to the results presented in Fig.10, Figs.11 to 13 display the dynamics of the ship under Roll motion. In Fig.11, the ship is rolled for 5 degrees. It can be seen that the Roll motion will be damped after couple of seconds and the Heave and Pitch motions due to the regular waves exhibit regular oscillations. Moreover, the values of acceleration in ship's bow and on the ship's center of gravity are presented. The values of acceleration also show regular oscillations similar to the ship motions. 
Table 2: Different experimental conditions for Model A.

\begin{tabular}{cccc}
\multicolumn{4}{c}{ Run Conditions } \\
\hline $\mathrm{NO}$ & $\mathrm{H} / \mathrm{b}$ & $\lambda /$ & $\mathrm{h} / \mathrm{H}$ \\
\hline 1 & 0.111 & 1 & 0.18 \\
2 & 0.167 & 1 & 0.17 \\
3 & 0.222 & 1 & 0.17 \\
4 & 0.056 & 2 & 0.93 \\
5 & 0.111 & 2 & 0.84 \\
6 & 0.167 & 2 & 0.81 \\
7 & 0.167 & 2 & 0.74 \\
8 & 0.222 & 2 & 0.73 \\
9 & 0.334 & 2 & 0.59 \\
10 & 0.334 & 2 & 0.61 \\
11 & 0.111 & 3 & 1.18 \\
12 & 0.222 & 3 & 1.13 \\
13 & 0.334 & 3 & 1.05 \\
14 & 0.111 & 4 & 1.23 \\
15 & 0.222 & 4 & 1.11 \\
16 & 0.334 & 4 & 1.07 \\
17 & 0.111 & 6 & 1.04 \\
18 & 0.334 & 6 & 0.97
\end{tabular}

(a)

(b)
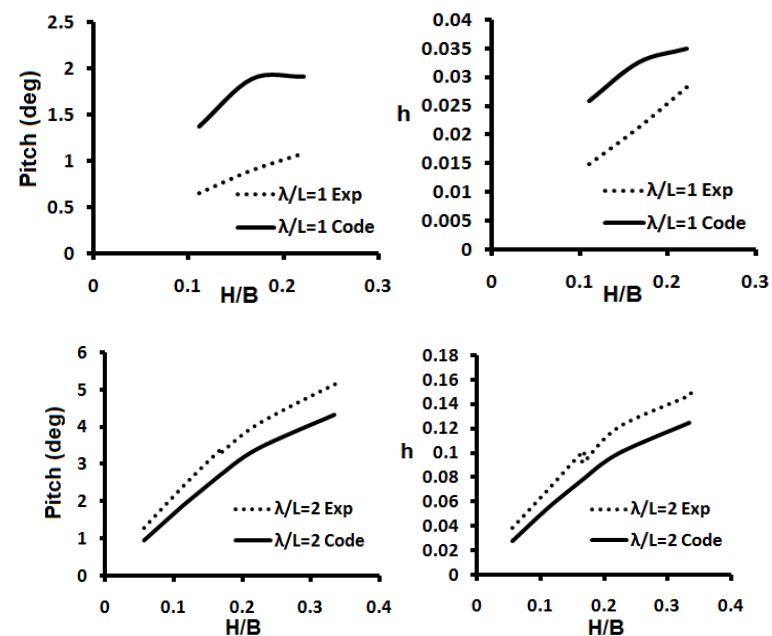

(c)
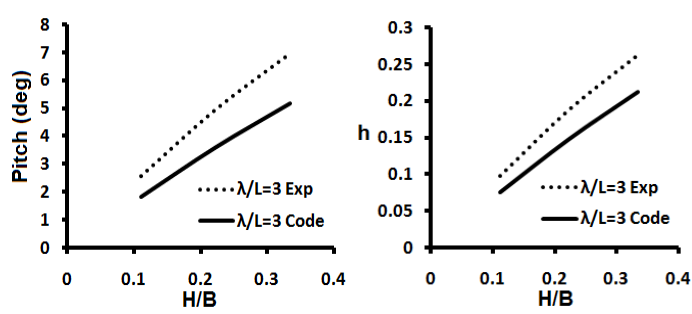
(d)
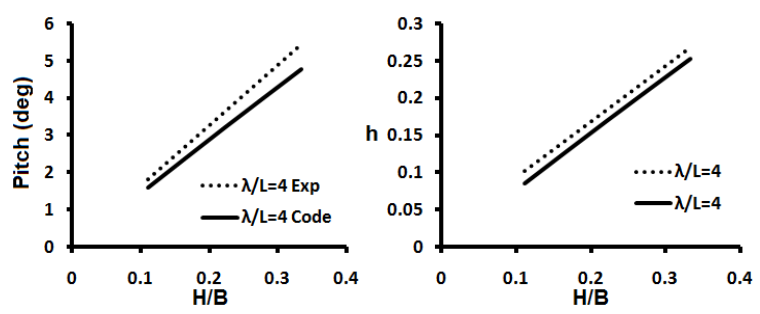

(e)
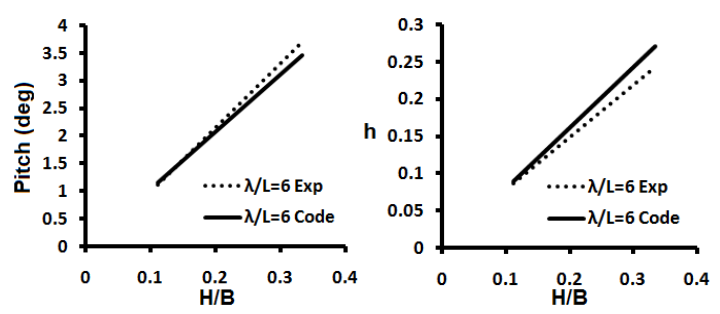

Fig.10: Comparison of the numerical results for Heave and Pitch motions and the experimental results in different wave lengths (Model A).
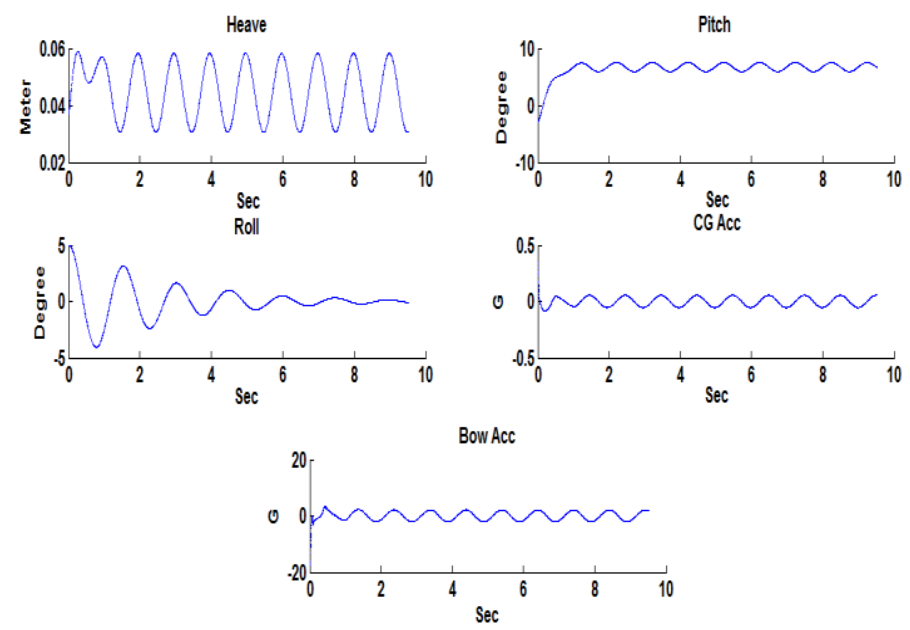

Fig.11: Planing vessel motions in 4 degrees of freedom (4-DOF) under regular waves.

Another studied case is for the dead-rise angle of 10 degrees. In this situation, as shown on Fig.12, reasonable results are obtained. It is worth mentioning that the Sway and Yaw motions of the ship are completely neglected and therefore the Roll motion is damped after a short while. If the Sway and Yaw motions were taken into consideration, considerable changes would be achieved.

Similar to the previous conditions, results for the dead-rise angle of 15 degrees can be studied. Generally, changes in the Roll angle would not have considerable effect on the Heave and Pitch motions unless the Yaw and Sway motions are taken into consideration.

\section{Conclusions}

Mathematical models that have been developed over the years are only able to analyze the ship's dynamic in 2 degrees of freedom. In the present paper, using the asymmetrical water impact of a wedge and 2D+t theory, a mathematical model is developed for the analysis of simultaneous Heave, Pitch and Roll motions. In addition to this, Momentum and Added Mass theories were utilized. The sea condition was modeled using regular waves. In this framework, experimental results are used for the validation of mathematical model. Results show that the present method is able to simulate the motions of the planing vessel with reasonable accuracy. Moreover, the ship motions are evaluated at three different initial Roll angles and it is shown that the vessel exhibits predictable behavior. In the next study, planing vessel's dynamic with consideration of Yaw and Sway motions will be analyzed. Also, it is desired to improve the accuracy of the model using different numerical techniques. 

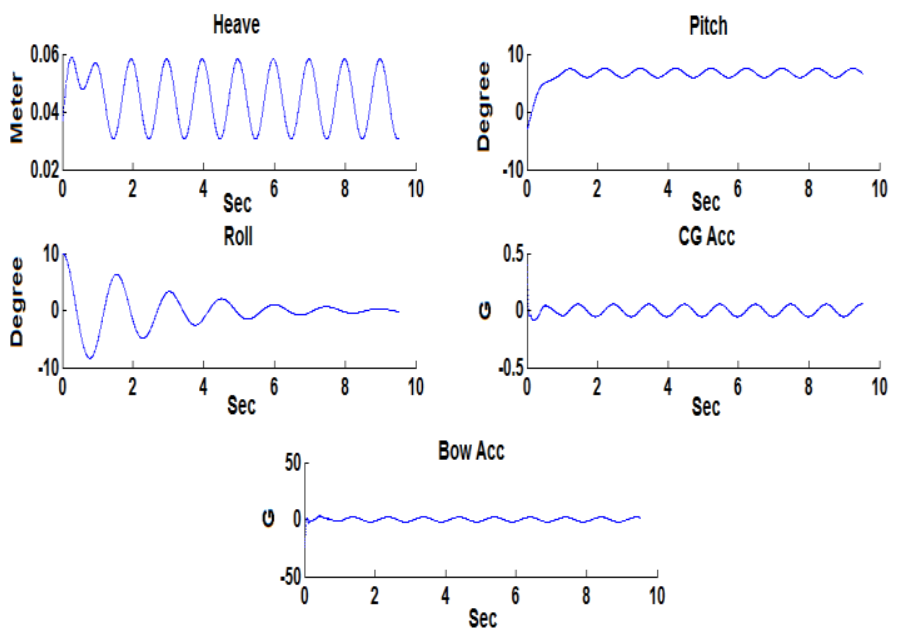

Fig.12: Planing vessel motions in 4 degrees of freedom (4-DOF) with initial Roll angle of 10 degrees.
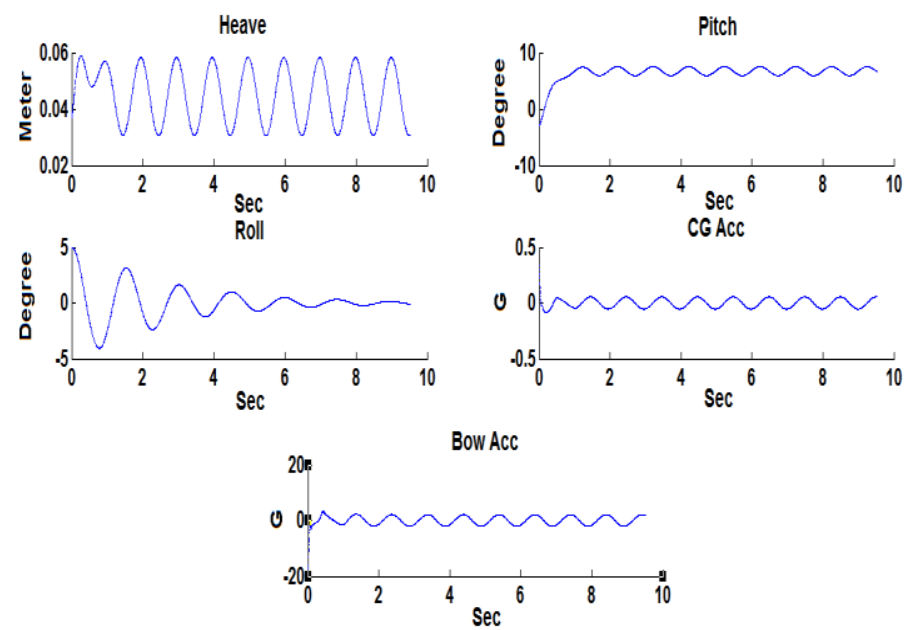

Fig.13: Planing vessel motions in 4 degrees of freedom (4DOF) with initial Roll angle of 15 degrees.

\section{References}

[1] Faltinsen, O. M., 2005, "Hydrodynamics of high-speed marine vehicles", Cambridge University Press

[2] Müller-Graf , B., 1997, "Dynamic stability of high speed small craft", WEGEMT Association, 25th School of craft technology, Atene, Greece

[3] Zhao, R. et al., 1997, "A simplified non-linear analysis of a high speed planing craft in calm water", 4th Int. Conf. On Fast Sea Transportation (FAST), Sydney, Australia

[4] Lai, C., \& Troesch, A. W., 1995, "Modeling issues related to the hydrodynamics of 3-dimensional steady planing”, Journal of Ship Research, Vol. 39, n. 1

[5] Martin, M., 1976, "Theoretical Predictions of Motions of High-Speed Planing Boats in Waves", David W. Taylor Naval Ship Research and Development Center, DTNSRDC 76/0069

[6] Zarnick, E., 1978, “A Nonlinear Mathematical Model of Motions of a Planing Boat in Regular, Waves”, David W. Taylor Naval Ship Research and Development Center, DTNSRDC 78/032

[7] Zarnick, E. 1979, "A Nonlinear Mathematical Model of Motions of a Planing Boat in Irregular Waves", DTNSRDC/SPD 0867-01

[8] Keuning, J. A., 1994, “The non-linear behaviour of fast mono-hulls in head waves”, PHD Thesis, Technische Universiteit Delft

[9] Payne, P., 1995, "Contribution to planing theory", Ocean Engineering Vol. 22 n. 7

[10] Garme, K., 2005, "Improved time domain simulation of planing hulls in waves by correction of the near transom lift", International Shipbuilding Progress, Vol. 52, n. 3 pp. 201-230

[11] Payne, P., 1995, "BOAT3D: a time-domain suite of computer programs for planing craft, version 2.3", Payne Associates, Severna Park, Maryland.

[12] Soleymannia, S.M., Ghadimi, P., Dashtimanesh, A., Feyz Chekab, A., "Analysis of the asymmetric wedge water impact" Iranian Journal of Marine Science and Technology, Noushahr, 2012.

[13] Wagner, H., "U. ber stoX-und Gleitvorga.nge an der Oberfla.che von Flu. ssigkeiten. Zeitschrift fr.", Angewandte Mathematik und Mechanik, 12, $192-215,1932$.

[14] Sebastianii, L., Bruzzone, D., Gualeni, P., "A practical method for the prediction of planing craft motions in regular and irregular waves" 2008 Proceedings of the 27th International Conference on Offshore Mechanics and Arctic Engineering, Estoril, 687-696, 2008.

[15] FRIDSMA, G., "A Systematic Study of the Rough-water Performanceof Planing Boats", Stevens Institute of Technology, Davidson Laboratory, Report 1275, November 1969 